\title{
Organ-specific expression of the divalent ion channel proteins NCKX3, TRPV2, CTR1, ATP7A, IREG1 and HEPH in various canine organs
}

\author{
CHANGHWAN AHN ${ }^{*}$, JONG-SAM CHOI ${ }^{*}$ and EUI-BAE JEUNG \\ Laboratory of Veterinary Biochemistry and Molecular Biology, College of Veterinary Medicine, \\ Chungbuk National University, Cheongju, Chungbuk 28644, Republic of Korea
}

Received December 2, 2017; Accepted May 3, 2018

DOI: $10.3892 / \mathrm{mmr} .2018 .9148$

\begin{abstract}
Transmembrane cation channels include those for calcium, copper and iron ion transport. Each channel has physiological significance, and all have been associated with disease. However, the comparative study of transcriptional-translational levels in canine organs has not been previously reported. In the present study, organ-specific expression of calcium channels, including sodium/potassium/calcium exchanger 3 (NCKX3) and transient receptor potential cation channel subfamily $\mathrm{V}$ member 2 (TRPV2), copper channels, including high affinity copper uptake protein 1 (CTR1) and copper-transporting ATPase 1 (ATP7A), and iron channels, including iron-regulated transporter 1 (IREG1) and hephaestin (HEPH) proteins and their mRNAs were examined in the canine duodenum, kidney, spleen and liver. NCKX3 protein expression was highest in the kidney, moderate in the duodenum, and lowest in the spleen and liver, whereas TRPV2 protein was highly expressed in the kidney, duodenum and liver, and was low in the spleen. The CTR1 protein expression level was highest in the liver, followed (in descending order) by the duodenum, kidney and spleen. The ATP7A protein expression level was highest in the duodenum and lowest in the spleen. The IREG1 protein expression level was highest in the liver, followed (in descending order) by the kidney, duodenum and spleen. The HEPH protein level was high in liver, moderate in the duodenum and kidney, and low in the spleen. The results of the immunohistochemistry analysis demonstrated ion
\end{abstract}

Correspondence to: Professor Eui-Bae Jeung, Laboratory of Veterinary Biochemistry and Molecular Biology, College of Veterinary Medicine, Chungbuk National University, 1 Chungdae Street, Building no. E12-1, Cheongju, Chungbuk 28644, Republic of Korea

E-mail: ebjeung@chungbuk.ac.kr

*Contributed equally

Key words: canine, divalent ion, gene expression channel protein localizations. These results suggested that cation channel proteins are differentially expressed among canine organs, and they may be involved in organ-specific functions associated with the maintenance of physiological homeostasis.

\section{Introduction}

Transmembrane cation channels include those for movement of calcium, copper, and iron ions (1). Calcium channels have selective permeability for calcium ions and regulate physiological responses associated with contraction of muscle, hormone and neurotransmitter release, activation of calcium-dependent enzymes, and calcium-dependent gene transcription (2). Copper channels transport copper ions into eukaryotic cells, bind copper ions, and exchange copper ions for intercellular components. Copper ions are important in the redox system as they act as cofactors for enzymes (3). Iron is notably involved in oxygen transport, the redox system and numerous metabolic enzymes. Transmembrane iron ion channels have roles in iron regulatory pathways and contribute to iron homeostasis (4).

The protein sodium/potassium/calcium exchanger 3 (NCKX3) is a $\mathrm{K}^{+}$-dependent $\mathrm{Na}^{+}-\mathrm{Ca}^{2+}$ exchanger and a member of solute carrier family 24 (5). The primary role of this exchanger is to control $\mathrm{Ca}^{2+}$ flux, through which it regulates intracellular $\mathrm{Ca}^{2+}$ homeostasis. NCKX3 is expressed in a number of organs and tissues, including the aorta and smooth muscle (6). Transcription of NCKX3 occurs principally in brain tissue, particularly in the thalamic nuclei, hippocampal CA1 neurons, and layer IV of the cerebral cortex (7). The calcium ion channel protein transient receptor potential cation channel subfamily $\mathrm{V}$ member 2 (TRPV2) is a member of the transient receptor potential (TRP) channel family (8). TRP channels serve to perceive various noxious mechanical, chemical and thermal stimuli. In particular, TRPV2 is specialized in the detection of thermal stimuli (9). A number of TRP channels regulate non-selective cation flux (including calcium and magnesium), and such flux may activate cellular signaling pathways (10). In addition, TRPV2 mediates calcium flow. TRPV2 is expressed in many parts of the immune system (including liver Kupffer cells, lung alveolar macrophages, mast cells and macrophages) 
and nervous system (including dorsal root ganglia and spinal motor neurons in developing mice) (11).

Copper transporter 1 (CTR1) is an adenosine 5'-triphosphate (ATP)-independent, high-affinity transporter, which is primarily present in the cell membrane (12). The high affinity of CTR1 for copper allows for an appropriate level of copper in the intracellular space. CTR1 is expressed in the majority of organs, and particularly in the liver, colon and intestine (13).

The copper-transporting ATPase 1 (ATP7A) copper transporter is a member of a family of P1B-type ATPases that transport heavy metals. ATP7A maintains copper homeostasis in conjunction with other copper transporters, including copper-transporting ATPase 2 (ATP7B) and CTR1 (14). ATP7A is present in a number of organs including kidney and placenta (15). If copper levels are elevated, ATP7A, which resides primarily in the trans-Golgi network, moves to the cell membrane in order to export excess copper from cells, prior to returning to its former position (16).

Iron-regulated transporter 1 (IREG1) is primarily detected in enterocytes and macrophages, and it has a role in iron efflux during intestinal iron absorption $(17,18)$. In addition, it has an iron recycling function in macrophages (19). IREG1 is a transmembrane protein that is regarded as a putative exporter of iron through the basolateral membrane to the plasma (20). The multicopper ferroxidase hephaestin (HEPH) converts $\mathrm{Fe}^{2+}$ into $\mathrm{Fe}^{3+}$ (21). HEPH is commonly expressed in supranuclear compartments and in the basolateral membranes of cells, including intestinal enterocytes (22). HEPH has a significant role in the transfer of dietary iron from enterocytes to blood, and is expressed in the intestinal tract, central nervous system, lung, pancreas and heart (23).

Cation channels are physiologically important and transmembrane channel dysfunction has been associated with a number of diseases. Impaired divalent ion homeostasis may induce neurodegenerative disorders including Alzheimer's and Parkinson's diseases, kidney stones, cardiac arrhythmia, anemia and hepatic disorders $(24,25)$. Thus, a number of investigations have been conducted into the expression and regulation of transmembrane cation channel proteins in a variety of organisms $(5,10,19,26-29)$. In this regard, investigations have been conducted to examine the expression and regulation of divalent ion channel proteins in experimental animals and humans (5,26,28,30-32). However, only a few studies have been performed using canine models. Tanner and Beeton (33) demonstrated that human channelopathies cannot fully be replicated in animal models, particularly mice or rats; however, canine models are quite similar to the human organ physiology $(34,35)$. In particular, comparisons between the mRNA and protein expression of divalent ion channels in canine organs have not been made. Thus, in the present study, the expression and localization of NCKX3, TRPV2, CTR1, ATP7A, IREG1 and HEPH proteins were investigated in canine organs. The organ-specific mRNA and protein expression of these factors was measured in the dog duodenum, kidney, spleen and liver by using reverse transcription (RT) polymerase chain reaction (PCR), RT-quantitative PCR (RT-qPCR), and western blot analyses. In addition, through immunohistochemical assessments, the localization of these molecules in canine organs was confirmed.

\section{Materials and methods}

Experimental animal model. A total of three 3-year-old intact female beagle dogs (average weight $20 \mathrm{~kg}$; purchased from Koatech, Pyeongtaek, Korea) were sacrificed. Prior to sacrifice, the dogs were fed with tap water and a commercial diet, ad libitum (Natural Balance Pet Foods, Inc., Burbank, CA, USA) and held in stainless steel cages. The cages were in a controlled environment maintained on a 12-h light/dark cycle (temperature, $23 \pm 2^{\circ} \mathrm{C}$; relative humidity, $50 \pm 10 \%$; ventilation, $17 \pm 1 \mathrm{times} / \mathrm{min})$. To collect samples from the duodenum, kidney, spleen and liver, the dogs were sacrificed with an injection of $\mathrm{KCl}$ and opened via a midline incision. All dissected organ samples were washed in cold sterile saline $(0.9 \% \mathrm{NaCl})$. All procedures for organ collection were approved by the Ethics Committee of Chungbuk National University (Cheongju, Republic of Korea).

Total RNA extraction, RT-PCR and $q P C R$. The whole organ samples were washed in cold sterile saline $(0.9 \% \mathrm{NaCl})$, placed in a volume of TRIzol ${ }^{\circledR}$ reagent (Invitrogen; Thermo Fisher Scientific, Inc., Waltham, MA, USA) that was proportional to the organ sample volume and homogenized in an ULTRA-TURRAX homogenizer (IKA-Works; Sigma-Aldrich; Merck KGaA, Darmstadt, Germany). RNA extraction from the homogenate was performed according to the manufacturer's protocol. The total RNA concentration was determined by measuring the absorbance at $260 \mathrm{~nm}$. Subsequently, RNA ( $1 \mu \mathrm{g})$ was transcribed using first-strand Moloney murine leukemia virus reverse transcriptase (Intron Biotechnology, Inc., Sungnam, Korea), a random 9-mer primer (Takara Bio, Inc., Otsu, Japan), and dNTPs (Takara Bio, Inc.) with first strand buffer (Thermo Fisher Scientific, Inc.) for $37^{\circ} \mathrm{C}$ for $1 \mathrm{~h}$ to synthesize cDNA. Random primers are composed of 9-mer deoxyribonucleotide mixtures, composed of a random sequence (up to $4^{9}$ different sequences) and a phosphorylated 5'-end.

For RT-PCR and RT-qPCR, $\beta$-actin was used as the endogenous reference for normalization and the determination of relative gene expression levels. The dog-specific primers were: NCKX3 sense, 5'-GGGCTCTGCAGTGTTCAATA-3' and antisense, 5'-GACTCCCACCAGGAAACTTG-3'; TRPV2 sense, 5'-GTGACTGGGGACTCCAT-3' and antisense, 5'-GAC CAGGAAGAGCAGTTCAAA-3'; CTR1 sense, 5'-CCA GGTTACCTCCTATTC-3' and antisense, 5'-TCATGTGCA TTCCCTCG-3'; ATP7A sense, 5'-CCCATAGCTGGA GTTTT-3' and antisense, 5'-TTCCGAAGGCCTTTTCTG TC-3'; IREG1 sense, 5'-GCCAGACTTAAAGTGGCT CA-3' and antisense), 5'-TGCAACATCGGCAATAGT GA-3'; HEPH sense, 5'-ACTGAAAGGGGTCAGGGTAA-3' and antisense, 5'-CCTTGGGAGCATAGTTCCAC-3'; and $\beta$-actin sense, 5'-AAGTCCAGCTTCTGTTTCCTC-3' and antisense, 5'-GCAGTGATCTCCTTCTGCAT-3'.

For RT-PCR, the NCKX3, TRPV2, CTR1, ATP7A, IREG1, HEPH, and $\beta$-actin were amplified in a PCR reaction $(20 \mu \mathrm{l})$ containing $1 \mathrm{U}$ i-StarTaq ${ }^{\mathrm{TM}}$ DNA polymerase (Intron Biotechnology, Inc.), $1.5 \mathrm{mM} \mathrm{MgCl} 2,2 \mathrm{mM}$ dNTP and $20 \mathrm{pmol}$ of the appropriate primers. PCR sequence parameters were 35 cycles of denaturation at $95^{\circ} \mathrm{C}$ for $30 \mathrm{sec}$, annealing at $57^{\circ} \mathrm{C}$ for $30 \mathrm{sec}$, and extension at $72^{\circ} \mathrm{C}$ for $30 \mathrm{sec}$. The obtained 
PCR products $(10 \mu \mathrm{l})$ were separated on $2.3 \%$ agarose gel and stained with ethidium bromide. Gel images were taken under ultraviolet illumination and were scanned using a Gel Doc EQ system (Bio-Rad Laboratories, Inc., Hercules, CA, USA).

For RT-qPCR, a $1-\mu 1$ amplicon of cDNA was assayed using SYBR (Takara Bio, Inc.) or TaqMan (Applied Biosystem; Thermo Fisher Scientific, Inc.) kits and following the manufacturers' protocols. The qPCR was performed using the following sequence: 30 cycles of denaturation at $95^{\circ} \mathrm{C}$ for $30 \mathrm{sec}$, annealing at $58^{\circ} \mathrm{C}$ for $30 \mathrm{sec}$ (and $60^{\circ} \mathrm{C}$ for $\mathrm{HEPH}$ ), and extension at $72^{\circ} \mathrm{C}$ for $30 \mathrm{sec}$. Data for each sample were analyzed by comparing cycle quantification $(\mathrm{Cq})$ values at a constant fluorescence intensity. The amount of transcript was inversely associated with the observed Cq, and for every two-fold dilution of the transcript the $\mathrm{Cq}$ was expected to increase by one increment. Relative expression $(\mathrm{R})$ was calculated as $\mathrm{R}=2^{-(\Delta \mathrm{Cq} \text { sample- } \Delta \mathrm{Cq} \text { control })}(36)$.

Western blot analysis. Whole organ samples obtained by sacrificing the mice, were washed in cold sterile saline $(0.9 \% \mathrm{NaCl})$, placed in $500 \mu \mathrm{l}$ or 1,000 $\mu \mathrm{l}$ Pro-prep (Intron Biotechnology, Inc.) depending on organ sample volume, and homogenized by using an ULTRA-TURRAX homogenizer. Protein samples were acquired from the suspension by centrifuging the homogenate at $17,800 \times \mathrm{g}$ at $4^{\circ} \mathrm{C}$ for $10 \mathrm{~min}$. Each $50-\mu \mathrm{g}$ sample was treated, in the following order, by mixing with SDS sample buffer, heating at $60^{\circ} \mathrm{C}$ for $10 \mathrm{~min}$, and centrifuging at $15,300 \mathrm{x} \mathrm{g}$ at $4^{\circ} \mathrm{C}$ for $3 \mathrm{~min}$. The $5-10 \% \mathrm{SDS}$ acrylamide gel was prepared and electrophoresis was accomplished. The gel was transferred to a polyvinylidene fluoride membrane (PerkinElmer, Inc., Waltham, MA, USA), and the membrane was blocked at room temperature for $1 \mathrm{~h}$ using $5 \%$ dry fat milk dissolved in TBS with Tween-20 (TBS-T). The membrane was incubated overnight at $4{ }^{\circ} \mathrm{C}$ with primary antibodies against the following factors: NCKX3 (cat. no. Sc-50129; 1:1,000; goat polyclonal), TRPV2 (cat. no. Sc-22520; 1:500; goat polyclonal; both Santa Cruz Biotechnology, Inc., Dallas, TX, USA), CTR1 (cat. no. NB100-402; 1:2,000; rabbit polyclonal; Novus Biologicals, LLC, Littleton, CO, USA), ATP7A (cat. no. Sc-32900; 1:2,000; rabbit monoclonal; Santa Cruz Biotechnology, Inc.), IREG1 (cat. no. ab85370; 1:2,000; rabbit polyclonal; Abcam, Cambridge, UK), HEPH (cat. no. ab56729, 1:2,000; mouse monoclonal; Abcam) and $\beta$-actin (cat no. Sc-130656; 1:1,000, mouse monoclonal, Santa Cruz Biotechnology, Inc.) diluted in bovine serum albumin (BSA; Sigma-Aldrich; Merck $\mathrm{KGaA}$ ). Subsequently, the membrane was washed four times for 7 min each with TBS-T. Secondary antibody conjugated with horseradish peroxidase (mouse polyclonal; cat. no. 7076S; or rabbit polyclonal; cat. no. $7074 \mathrm{~S}$; both Cell Signaling Technology, Inc., Danvers, MA, USA; or goat polyclonal; cat. no. sc-2004; Santa Cruz Biotechnology, Inc.) diluted 2,000-fold in $2.5 \%$ non-fat dry milk dissolved in TBS-T for $1 \mathrm{~h}$ at room temperature. The membrane was washed as described above. Following washing, the blots were developed by incubation with an enhanced chemiluminescence reagent (Merck KGaA) and exposed to Biomax ${ }^{\mathrm{TM}}$ Light Film (Kodak, Rochester, NY, USA) for 1-5 min. Signal specificity was confirmed by blotting without a primary antibody. Experimental bands were normalized to those of $\beta$-actin. The signal intensity of each band was measured using ImageJ software (v 1.50b; National Institutes of Health, Bethesda, MD, USA). To determine mean band density, the background signal from an area near each lane was subtracted from each band.

Immunohistochemistry. Theorgan-specific localization of cation channels was investigated by performing immunohistochemical assessment. The canine duodenum (upper part of the duodenum, $3 \mathrm{~cm}$ from gastric pylorus), kidney (borderline of the cortex and medulla), spleen (borderline of the white and red pulp) and liver (quadrate lobe of the liver) were examined. Each part of the tissue (duodenum, kidney, spleen and liver) was identified by macroscopic necropsy. The excised organ samples (duodenum, kidney, spleen and liver) were immediately washed in sterile saline. Tissues were processed with $70 \%$ ethanol for $1 \mathrm{~h}$, $95 \%$ ethanol (95\% ethanol/5\% methanol) for $1 \mathrm{~h}$, pure ethanol for $1 \mathrm{~h}$, a second time with pure ethanol for $90 \mathrm{~min}$, a third time with pure ethanol for $90 \mathrm{~min}$, a fourth time with pure ethanol for $2 \mathrm{~h}$, twice with clearing agent for $1 \mathrm{~h}$, twice with wax at $58^{\circ} \mathrm{C}$ for $1 \mathrm{~h}$, and embedded in paraffin. Embedded blocks were sectioned to $4 \mu \mathrm{m}$, and the sections were mounted on glass slides (Muto Pure Chemicals, Tokyo, Japan) precoated with aminosilane, deparaffinized using xylene, and hydrated in a descending concentration gradient of ethanol solutions (100, 95, 75 and 60\%; 5 min for each solution). Subsequently, the slide was boiled in antigen retrieval solution buffered with Tris for $20 \mathrm{~min}$, cooled at room temperature for $30 \mathrm{~min}$, and washed with TBS-T for $5 \mathrm{~min}$. To block endogenous peroxidase, the slide was placed in $3 \%$ hydrogen peroxide for $30 \mathrm{~min}$ at room temperature and washed three times for $10 \mathrm{~min}$ each with TBS-T. To avoid non-specific reactions, the sections were incubated with 1X BSA in PBS for $30 \mathrm{~min}$ at room temperature, followed by washing three times for 10 min each with TBS-T. Following washing, the slides were incubated with primary antibodies (as detailed above for western blotting) overnight at room temperature in a moist chamber. Each primary antibody was diluted 250-fold in BSA. Slides were rinsed with TBS-T three times for $10 \mathrm{~min}$ each to remove antibody. Biotinylated secondary antibodies [1:500; Goat (cat. no. BA-9500), Mouse (cat. no. BA-9200) or Rabbit (cat. no. BA-1000) IgG; Vector Laboratories, Ltd., Peterborough, UK] were added and incubated at $37^{\circ} \mathrm{C}$ for $30 \mathrm{~min}$. Slides were washed following the incubation with the primary antibody. Subsequently, ABC Elite (Vector Laboratories, Ltd.) was added to the slides, which were incubated at $37^{\circ} \mathrm{C}$ for $1 \mathrm{~h}$ and washed three times for $10 \mathrm{~min}$ each with TBS-T. Diaminobenzidine (DAB; Vector Laboratories, Ltd.) was used as a chromogen. Slides were incubated with DAB at room temperature for $20 \mathrm{sec}$. Counterstaining was achieved using Harris hematoxylin at room temperature for $20 \mathrm{sec}$ (Sigma-Aldrich; Merck KGaA). Images of the tissue were obtained using a light microscope (BX51 Standard Microscope; Olympus, Tokyo, Japan; magnification, x200 and $\mathrm{x} 400)$.

Data analysis. The purpose of the present study was to present the distribution of divalent ion channel mRNA/protein expression and localization in canine organs, including the duodenum, kidney, spleen and liver. Therefore, data are presented as the mean \pm standard deviation. Each experiment was repeated three times. Statistical analyses were performed using GraphPad Prism software v4.0 (GraphPad Software, Inc., La Jolla, CA, USA). 
A

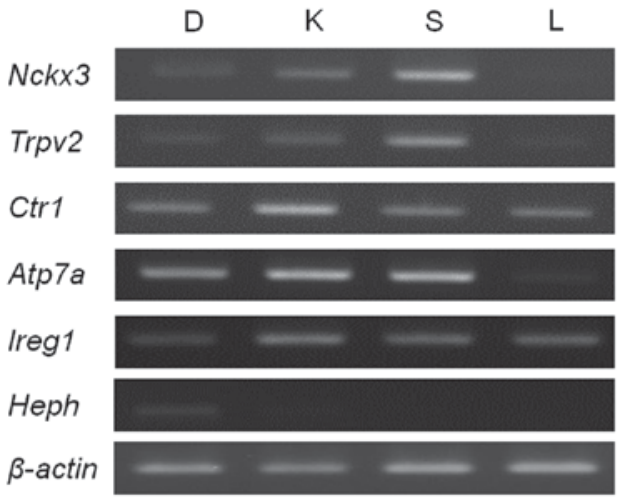

B

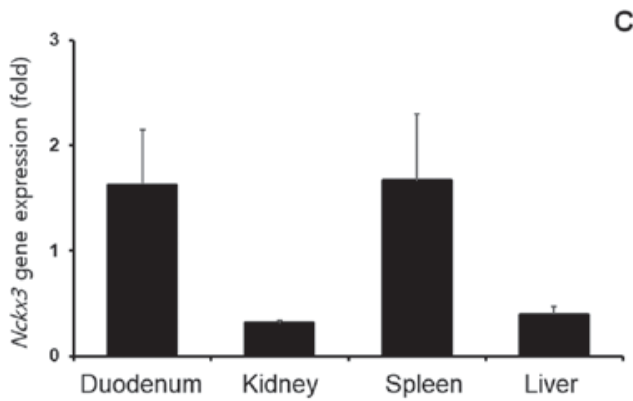

D

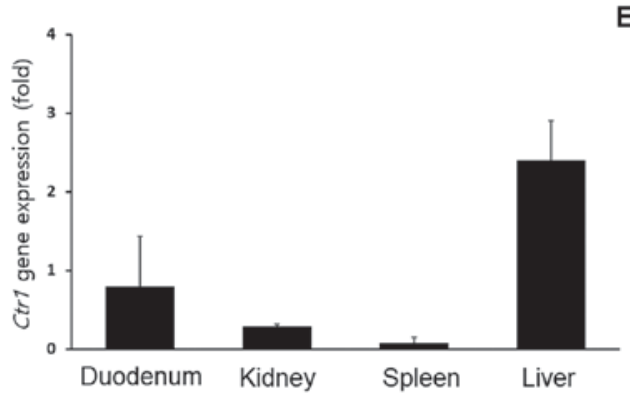

$\mathrm{C}$

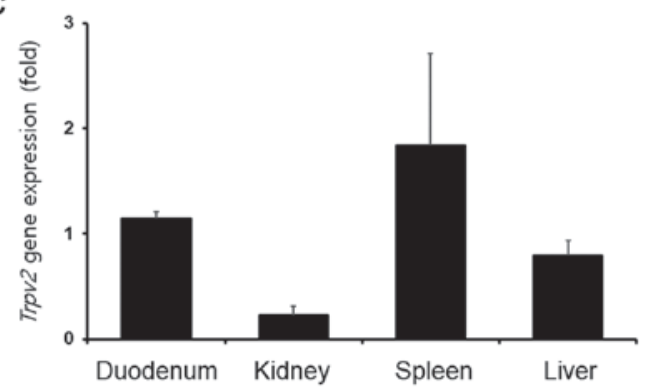

E

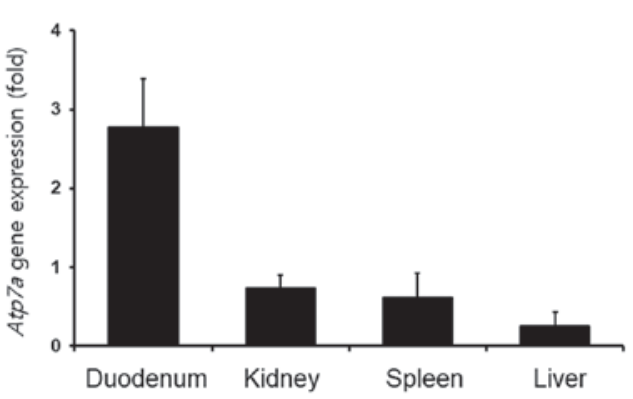

$\mathbf{F}$

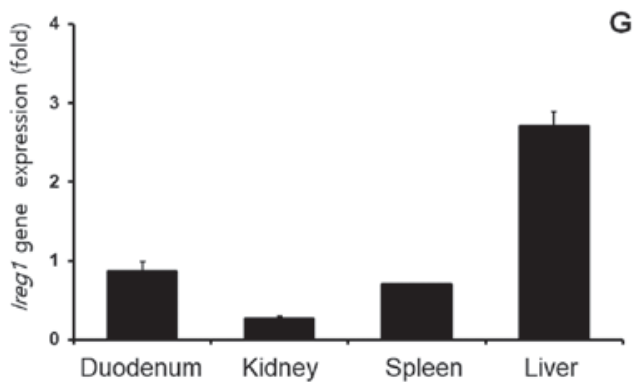

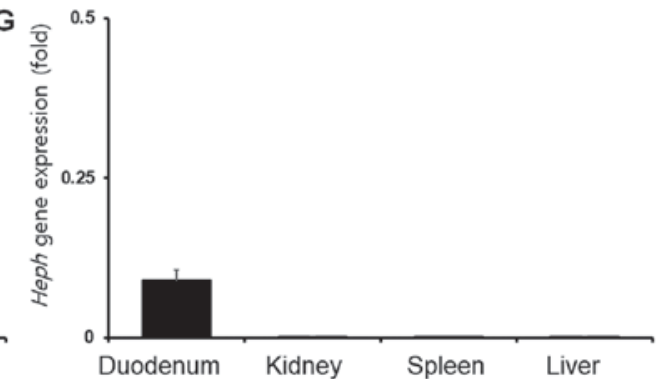

Figure 1. Organ-specific mRNA expression of NCKX3, TRPV2, CTR1, ATP7A, IREG1 and HEPH. (A) Representative image of relative expression, and quantification of (B) NCKX3, (C) TRPV2, (D) CTR1, (E) ATP7A, (F) IREG1 and (G) HEPH in the canine duodenum, kidney, spleen and liver, as measured by RT-qPCR. D, duodenum; K, kidney; S, Spleen; L, Liver; ATP7A, copper-transporting ATPase 1; CTR1, copper uptake protein 1; HEPH, hephaestin; IREG1, iron-regulated transporter 1; NCKX3, sodium/potassium/calcium exchanger 3; TRPV2, transient receptor potential cation channel subfamily V member 2.

\section{Results}

Organ-specific mRNA expression of canine divalent ion channels. Organ-specific mRNA expression of NCKX3 in the canine duodenum, kidney, spleen and liver was analyzed by performing RT-PCR and qPCR (Fig. 1A and B) with $\beta$-actin as an internal control. The results demonstrated that NCKX3 mRNA levels were high in the duodenum and spleen, and low in the kidney and liver. The RT-PCR and qPCR results for TRPV2 mRNA expression levels were different among the sampled organs (Fig. 1C). The TRPV2 mRNA expression was high in the spleen, moderately high in the duodenum and liver, and low in the kidney. The expression of CTR1 varied among the sampled organs. CTR1 mRNA expression levels were the highest in the liver (Fig. 1D) followed, in descending order, by the duodenum, kidney and spleen. The ATP7A mRNA levels were highest in the duodenum (Fig. 1E) followed, in descending order, by the kidney, spleen and liver. IREG1 mRNA expression was high in the liver, moderately high in the duodenum and spleen, and low in the kidney (Fig. 1F). Finally, HEPH mRNA expression levels were notably low in the duodenum and almost undetectable in the kidney, spleen and liver (Fig. 1G). 
A

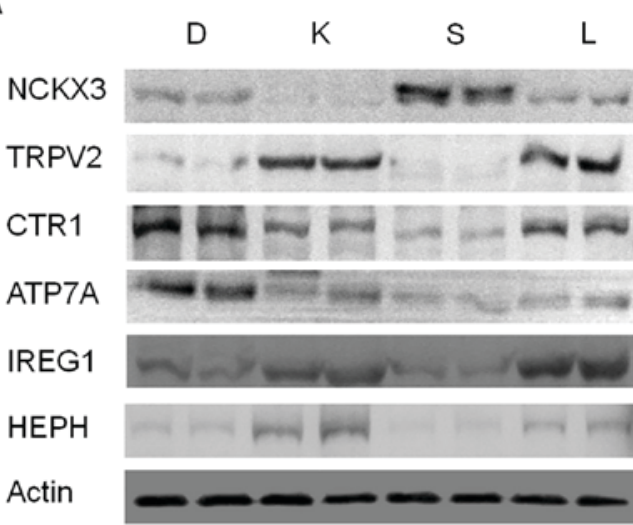

B

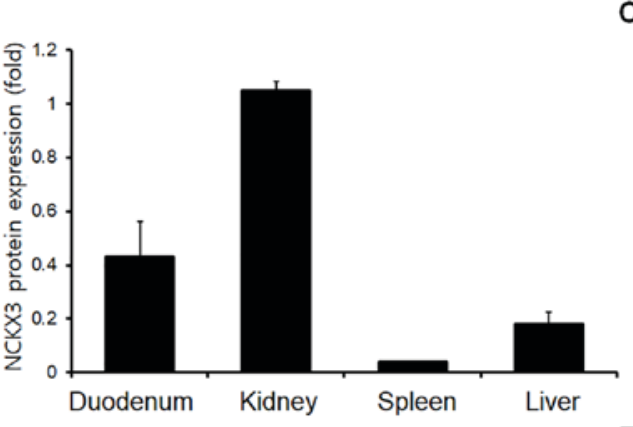

C

D

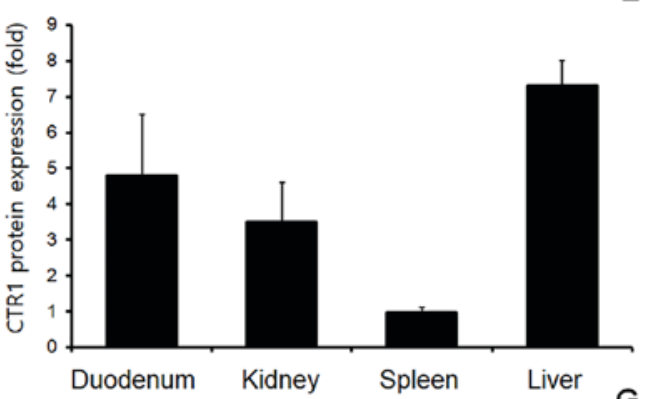

E
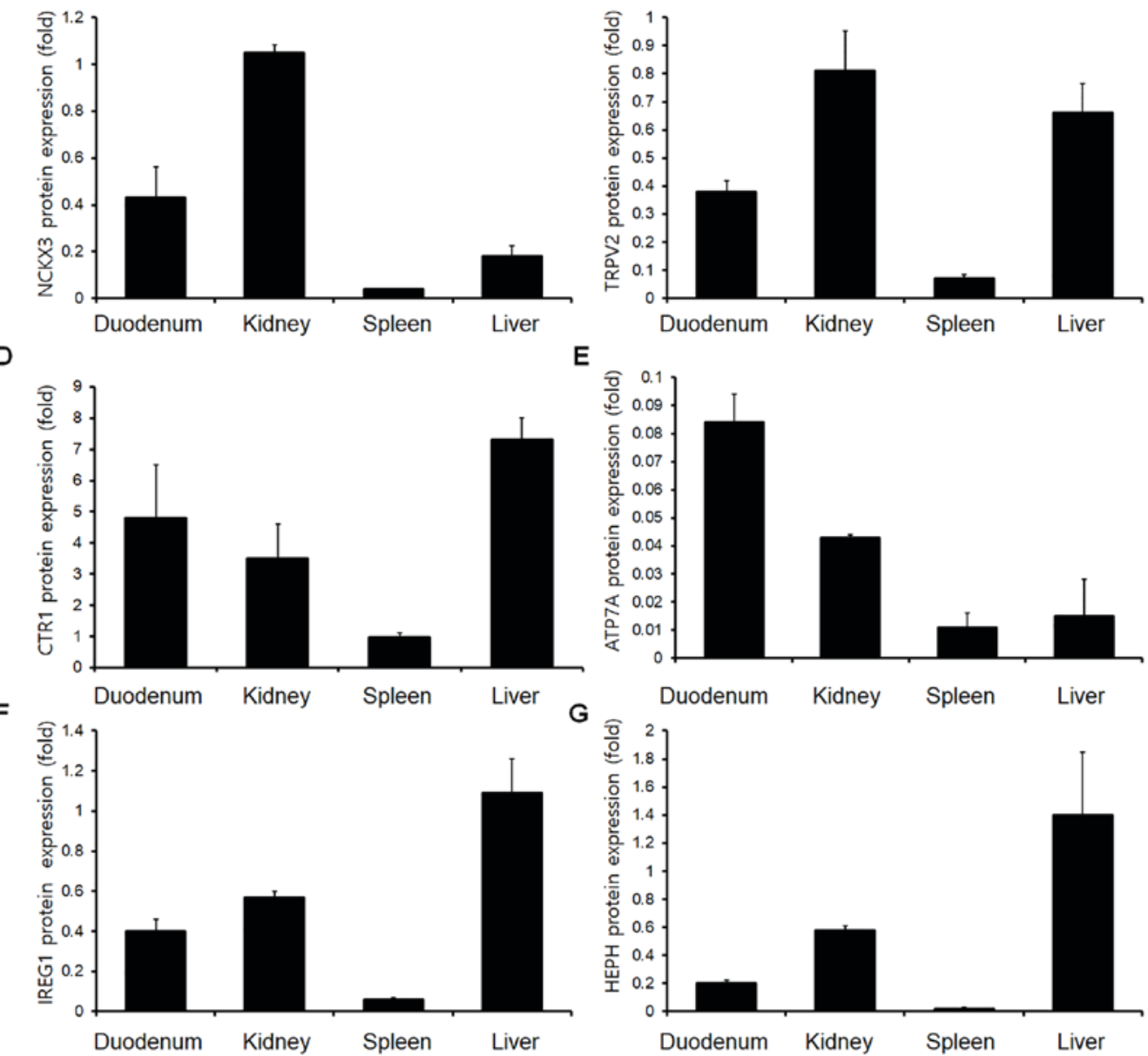

Figure 2. Western blot analysis of NCKX3, TRPV2, CTR1, ATP7A, IREG1 and HEPH. (A) Representative western blotting images and quantification of (B) NCKX3, (C) TRPV2, (D) CTR1, (E) ATP7A, (F) IREG1 and (G) HEPH protein expression levels in the canine duodenum, kidney, spleen and liver. D, duodenum; K, kidney; S, Spleen; L, Liver; ATP7A, copper-transporting ATPase 1; CTR1, copper uptake protein 1; HEPH, hephaestin; IREG1, iron-regulated transporter 1; NCKX3, sodium/potassium/calcium exchanger 3; TRPV2, transient receptor potential cation channel subfamily V member 2.

Organ-specific protein expression of canine divalent ion channels. Western blot analysis was performed to identify canine ion channel protein expression in the duodenum, kidney, spleen and liver (Fig. 2A). The NCKX3 protein expression was highest in the kidney, moderate in the duodenum, and low in the spleen and liver (Fig. 2B). The TPRV2 protein expression levels in the various organs were different from the mRNA levels (Fig. 2C). In the kidney, duodenum and liver, TRPV2 protein was highly expressed, whereas its expression was low in the spleen. The CTR1 protein expression levels were similar to the mRNA results, as its expression was the highest in the liver, followed, in descending order, by the duodenum, kidney, and spleen (Fig. 2D). Compared with the other transmembrane cation channel factors examined, the ATP7A protein expression levels were relatively low in all organs (Fig. 2E). Similarly, the ATP7A protein expression levels were the highest in duodenum; however, the lowest expression level was in the spleen. Western blotting results demonstrated that the IREG1 protein expression level was the highest in the liver followed, in descending order, by the kidney, duodenum and 

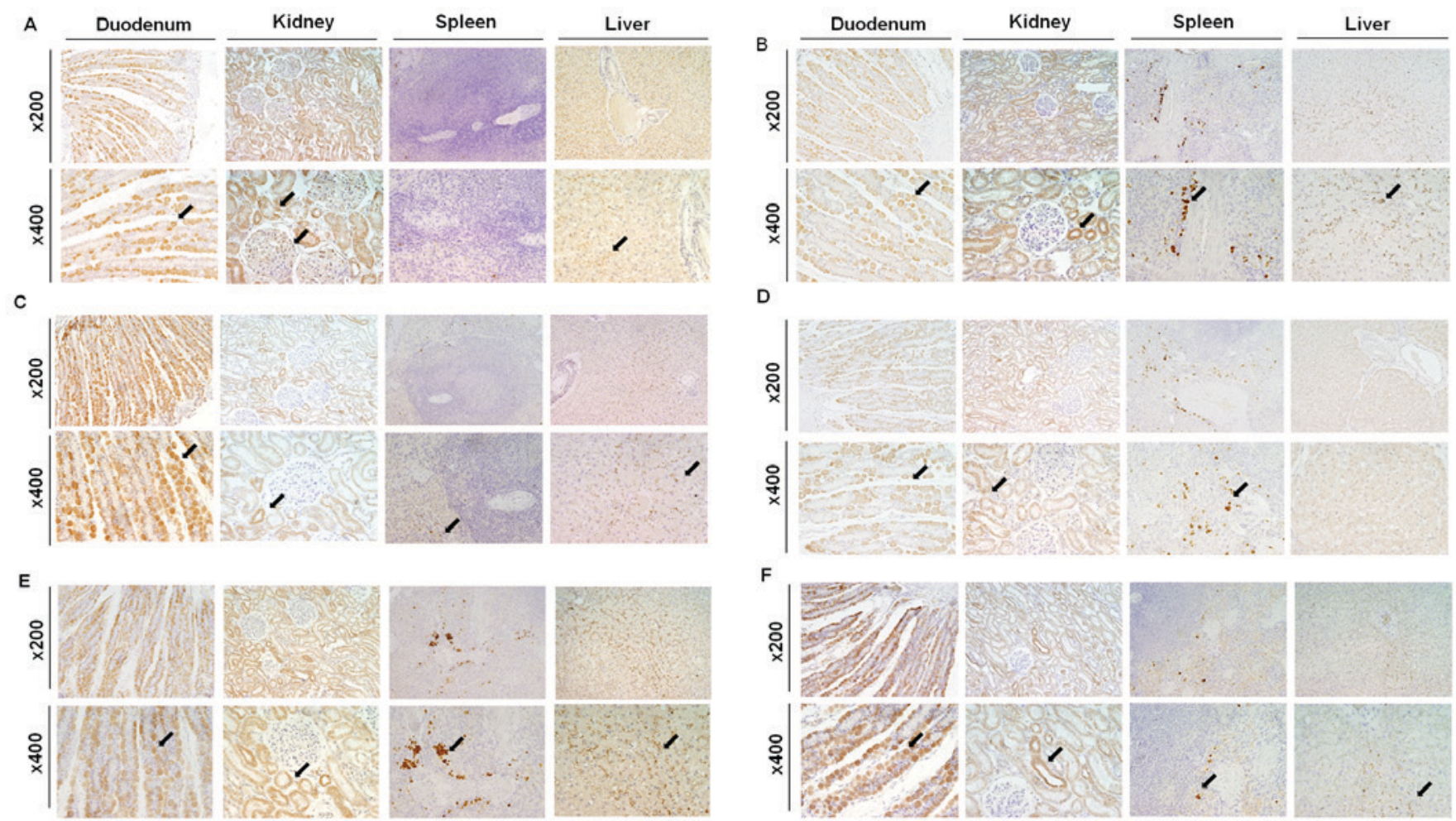

F
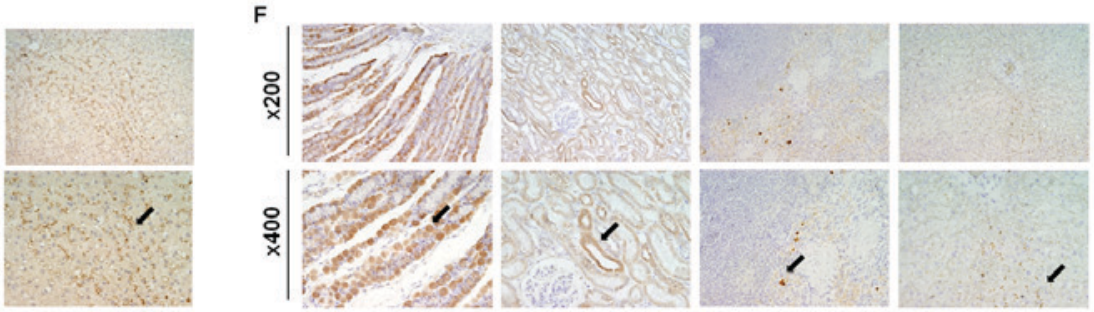

Figure 3. Immunohistochemistry of NCKX3, TRPV2, CTR1, ATP7A, IREG1 and HEPH. Organ-specific localization of (A) NCKX3, (B) TRPV2, (C) CTR1, (D) ATP7A, (E) IREG1 and (F) HEPH in the canine duodenum, kidney, spleen and liver. Magnification, $\mathrm{x} 200$ and $\mathrm{x} 400$. Black arrows indicate the immunopositive sites in each tissue. ATP7A, copper-transporting ATPase 1; CTR1, copper uptake protein 1; HEPH, hephaestin; IREG1, iron-regulated transporter 1; NCKX3, sodium/potassium/calcium exchanger 3; TRPV2, transient receptor potential cation channel subfamily V member 2.

spleen (Fig. 2F). The HEPH protein expression level results demonstrated a high level in the liver, moderately high levels in the duodenum and kidney, and a low level in spleen (Fig. 2G).

Organ-specific localization of canine divalent ion channels. Organ-specific localization of cation channels was investigated by immunohistochemistry (Fig. 3). Immunohistochemical analysis revealed that NCKX3 was expressed in the intestinal villi and hepatocytes, in addition to tubules, glomeruli, and Henle's loops of the kidney (Fig. 3A). Immunohistochemical analysis demonstrated that TRPV2 was present in all sampled organs (Fig. 3B) and was detected in intestinal villi, convoluted tubules and Henle's loops in the kidney, red pulp and macrophages in the spleen, and Kupffer cells in the liver. In the magnified images, certain cells in the spleen, which contained numerous nuclei were stained with DAB and consequently identified as splenic macrophages. The CTR1 immunohistochemical staining results demonstrated its presence in all sampled organs (Fig. 3C). In the duodenum, CTR1 was present in intestinal villi. In addition, it was observed in distal convoluted tubules in the kidney, red pulp in the spleen, and canaliculi in the liver. The immunohistochemical staining results demonstrated that ATP7A was expressed in intestinal villi, tubules in the kidney, and macrophages in the marginal zone of spleen (Fig. 3D). The immunohistochemical staining results demonstrated that IREG1 was present in intestinal villi, the proximal and distal convoluted tubules in the kidney, macrophages in the spleen, and Kupffer cells in the liver (Fig. 3E). Finally, the HEPH immunohistochemical staining results demonstrated that $\mathrm{HEPH}$ protein was present in intestinal villi, the distal convoluted tubules in the kidney, macrophages in the spleen, and hepatocytes and Kupffer cells in the liver (Fig. 3F).

\section{Discussion}

Cation transmembrane channels, including those for calcium, copper and iron ions, have important roles in the bodies of humans and animals (5). The functions of these channels are directly connected with the health of the organism and include roles in homeostasis and metabolism $(5,31,37)$. All aspects of metabolism, including hormone secretion, vitamin synthesis, food digestion and the absorption of nutrients are regulated via cation transmembrane channels (38). These channels are located in a variety of organs and have the ability to function at metabolically appropriate times (39). A number of previous studies have reported on the expression and regulation of various cation channel proteins; however, there are no similar reports for such proteins in canine organs $(2,31,39-41)$. Thus, in the present study, the organ-specific expression and localization of calcium (NCKX3, TRPV2), copper (CTR1, ATP7A), iron (IREG1) and ferroxidase for iron transporting (HEPH) proteins and mRNAs were investigated in the canine duodenum (upper part of the duodenum), kidney (borderline of the cortex and medulla), spleen (borderline of the white and red pulp) and liver (quadrate lobe of the liver). Each tissue (duodenum, kidney, spleen and liver) is composed of various cell types, resulting in heterogeneity in the samples. Therefore, immunohistochemistry was performed to identify 
the tissue-specific expression of divalent ion channel proteins to address this limitation.

The expression of NCKX3 in the mouse kidney is reported to be different between males and females, with the female NCKX3 expression level being notably higher than that in the male mouse (27). Through the action of NCKX3, intracellular potassium and calcium ions are exchanged for four extracellular sodium ions. In addition, it has been reported that NCKX3 is important in calcium reabsorption in the kidney in the presence or absence of another calcium-associated gene activity (27). In the present results, NCKX3 protein was highly expressed in the canine kidney and was predominantly localized in distal convoluted tubules, with moderately high localization in the proximal convoluted tubules. In the kidney, NCKX3 in the renal tubular epithelium has a key role in calcium reabsorption (6). In another study, NCKX3 was demonstrated to be expressed in the rat duodenum (27). NCKX3 in the basolateral membrane of intestinal tissue transports calcium to the blood, thus it has a key role in calcium absorption (41). Due to this function of NCKX3, abnormal expression of NCKX3 can induce decreased calcium absorption in the duodenum and calcium reabsorption in the kidney; furthermore, it may result in hypocalcemia and osteoporosis (42). In the present study, the results demonstrated that NCKX3 mRNA levels were high in the duodenum, spleen and kidney, and low in the liver. Based on these results and those from previous studies, NCKX3 may have an important role in maintaining calcium homeostasis in the canine duodenum, spleen and kidney for preventing hypocalcemia and osteoporosis.

The results of previous studies have demonstrated that TRPV2 has a key role in macrophage phagocytosis, and one study revealed that TRPV2 is involved in the earliest stages of phagocytosis (37,43-45). In addition, TRPV2 functions in intra- and extracellular $\mathrm{Ca}^{2+}$ mobilization, and it has a role in lipopolysaccharide-induced cytokine production in macrophages (46). The present results demonstrated that TRPV2 was present in the liver and spleen macrophages. Another study demonstrated that TRP family members are expressed in the gastrointestinal tract (31) and kidney, and they have roles in mucosal function and $\mathrm{Ca}^{2+}$ homeostasis (47). TRPV2 also has a notable immunomodulatory function (48), and abnormalities in its expression create issues, including the development of chemoattractant-elicited, motility-defective macrophages (40). Based on the present results and those of previous studies, TRPV2 may have an important role in macrophage phagocytosis and cytokine production in dogs. In addition, TRPV2 may be hypothesized to have a function in $\mathrm{Ca}^{2+}$ homeostasis in dogs.

CTR1 protein expression levels in the present study were high in the duodenum, kidney, and liver, and low in the spleen. A previous study reported that CTR1 expression was high in liver canaliculi, kidney convoluted tubules, and intestinal enterocytes in mice (30). In general, CTR1 regulates copper levels between the intra- and extracellular spaces, in order to maintain homeostatic balance on the two sides of the cell membrane (36-38). In the intestine, CTR1 has been demonstrated to have an important role in copper absorption and regulation. Furthermore, it was revealed that CTR1 is expressed primarily on the apical surface of intestinal epithelial cells (28). Another study indicated that the liver is an important organ for copper storage, as mouse hepatocytes lacking CTR1 had a low copper concentration and their copper-dependent hepatic enzymes had reduced activity (49). In addition, a study suggested that CTR1 has a critical role in copper reabsorption in the kidney, since mice lacking CTR1 exhibited a high copper concentration in the urine (12). These results indicated that CTR1 may have a significant role in copper absorption and homeostatic regulation in the canine duodenum and kidney. Copper storage and liver enzyme activity may be hypothesized to be influenced by CTR1 expression in dogs.

A previous study demonstrated that ATP7A expression in the intestine has a copper efflux function following dietary copper absorption (50). Another study demonstrated that ATP7A is expressed in the kidney and contributes to renal copper homeostasis by acting in conjunction with ATP7B. Furthermore, ATP7A has been demonstrated to have a compensatory copper export function in order to maintain renal copper homeostasis in ATP7B-lacking mice (51). Silencing of ATP7A has resulted in attenuated macrophages, thus indicating its presence in macrophages; additionally, ATP7A has weak bactericidal activity (29). In addition, ATP7A has been associated with certain diseases, including Menke's syndrome. The ATP7A mutation that results in Menke's syndrome is an X-linked recessive disorder associated with copper deficiency, resulting in reduced functioning of copper-dependent enzymes (52). Based on these observations and those in the present study, ATP7A may have important roles in copper homeostasis and immunological function in dogs.

Previous studies have demonstrated that IREG1 protein, a principal modulator of iron homeostasis, is expressed in the rat duodenum, liver and reticuloendothelial system macrophages $(19,20)$. It has also been reported that IREG1 protein is located in the basolateral plasma membrane of intestinal cells and acts as an iron exporter $(19,20)$. Furthermore, IREG1 is expressed in the medullary portions of nephrons and proximal tubules (32). Similarly, the results of the present study demonstrated that IREG1 is expressed in canine kidney tubules and intestinal cells. In the kidney, IREG1 is active in exporting iron when iron levels increase. Mutations of IREG1 may result in iron overload, as exhibited in type IV hemochromatosis $(17,19,20)$. In addition, if there is an imbalance between IREG1 and hepcidin levels, systemic iron homeostasis is lost, which may result in tumors including breast cancer (53). Based on these results, IREG1 is hypothesized to function as an iron exporter and in the maintenance of iron homeostasis in dogs.

HEPH presence has been reported in intestinal tissue, particularly in the basolateral membrane of human enterocytes (26). In rats, HEPH has been located in the duodenum, and, in conjunction with other iron-associated proteins including hepcidin, is reported to be active in the maintenance of iron homeostasis (54). The present results demonstrated that HEPH mRNA levels were higher in the canine duodenum compared with other organs. In addition, immunohistochemical staining results demonstrated a marked presence of HEPH in duodenal enterocytes. Based on results from other animal studies, it appears that HEPH protein in the canine duodenum may have a notable role in transporting dietary iron from enterocytes to the circulatory system $(21-23,26,54,55)$. Other studies have reported HEPH presence in the rat liver (56) and in the mouse spleen and kidney (55). HEPH mutation has been associated 
with systemic iron deficiency, and an absence of HEPH may result in iron accumulation in duodenal enterocytes, resulting in microcytic and hypochromic anemia in mouse (23). Based on the present results and those of other studies, HEPH is hypothesized to have an important role in maintaining iron homeostasis and preventing diseases associated with iron imbalance in dogs.

In conclusion, the present results demonstrated that NCKX3, TRPV2, CTR1, ATP7A, IREG1 and HEPH protein and mRNA are expressed and localized in a tissue-specific manner in canines. These results suggest that such transmembrane cation proteins have characteristic functions in the tissues in which they are highly expressed. The results from the present study provide a basis for further studies into transmembrane cation channel proteins and their functions in canine pathological and physiological states.

\section{Acknowledgements}

Not applicable.

\section{Funding}

The present study was supported by a National Research Foundation of Korea (grant no. 2017R1A2B2005031) grant funded by the Korean government and the Global Research and Development Center Program through the National Research Foundation of Korea, funded by the Ministry of Education, Science and Technology (grant no. 2017K1A4A3014959).

\section{Availability of data and materials}

All data generated or analyzed during this current study are included in this published article.

\section{Authors' contributions}

E-BJ designed the study and analyzed the data. CA and J-SC performed RNA/protein experiments and interpreted the data. CA, J-SC and E-BJ wrote the manuscript. All authors read and approved the final manuscript.

\section{Ethics approval and consent to participate}

All procedures for organ collection were approved by the Ethics Committee of Chungbuk National University (Cheongju, Republic of Korea).

\section{Consent for publication}

Not applicable.

\section{Competing interests}

The authors declare that they have no competing interests.

\section{References}

1. Argüello JM, Raimunda D and González-Guerrero M: Metal transport across biomembranes: Emerging models for a distinct chemistry. J Biol Chem 287: 13510-13517, 2012.
2. Turner RW, Anderson D and Zamponi GW: Signaling complexes of voltage-gated calcium channels. Channels (Austin) 5: 440-448, 2011.

3. Maryon EB, Molloy SA, Ivy K, Yu H and Kaplan JH: Rate and regulation of copper transport by human copper transporter 1 (hCTR1). J Biol Chem 288: 18035-18046, 2013.

4. Anderson ER and Shah YM: Iron homeostasis in the liver. Compr Physiol 3: 315-330, 2013.

5. Jalloul AH, Szerencsei RT and Schnetkamp PP: Cation dependencies and turnover rates of the human $\mathrm{K}^{+}$-dependent $\mathrm{Na}^{+}-\mathrm{Ca}^{2+}$ exchangers NCKX1, NCKX2, NCKX3 and NCKX4. Cell Calcium 59: 1-11, 2016.

6. Yang H, Lee GS, Yoo YM, Choi KC and Jeung EB: Sodium/potassium/calcium exchanger 3 is regulated by the steroid hormones estrogen and progesterone in the uterus of mice during the estrous cycle. Biochem Biophys Res Commun 385: 279-283, 2009.

7. Kraev A, Quednau BD, Leach S, Li XF, Dong H, Winkfein R, Perizzolo M, Cai X, Yang R, Philipson KD and Lytton J: Molecular cloning of a third member of the potassium-dependent sodium-calcium exchanger gene family, NCKX3. J Biol Chem 276: 23161-23172, 2001.

8. Nilius B and Owsianik G: The transient receptor potential family of ion channels. Genome Biol 12: 218, 2011.

9. Park DJ, Kim SH, Nah SS, Lee JH, Kim SK, Lee YA, Hong SJ, Kim HS, Lee HS, Kim HA, et al: Polymorphisms of the TRPV2 and TRPV3 genes associated with fibromyalgia in a Korean population. Rheumatology (Oxford) 55: 1518-1527, 2016.

10. Kunert-KeilC,BispingF,KrugerJand BrinkmeierH:Tissue-specific expression of TRP channel genes in the mouse and its variation in three different mouse strains. BMC Genomics 7: 159, 2006.

11. Perálvarez-Marín A, Doñate-Macian P and Gaudet R: What do we know about the transient receptor potential vanilloid 2 (TRPV2) ion channel? FEBS J 280: 5471-5487, 2013.

12. Tsai CY, Liebig JK, Tsigelny IF and Howell SB: The copper transporter 1 (CTR1) is required to maintain the stability of copper transporter 2 (CTR2). Metallomics 7: 1477-1487, 2015.

13. Landon CD, Benjamin SE, Ashcraft KA and Dewhirst MW: A role for the copper transporter Ctr1 in the synergistic interaction between hyperthermia and cisplatin treatment. Int $\mathrm{J}$ Hyperthermia 29: 528-538, 2013.

14. Yi L and Kaler SG: Direct interactions of adaptor protein complexes 1 and 2 with the copper transporter ATP7A mediate its anterograde and retrograde trafficking. Hum Mol Genet 24: 2411-2425, 2015.

15. La Fontaine S and Mercer JF: Trafficking of the copper-ATPases, ATP7A and ATP7B: Role in copper homeostasis. Arch Biochem Biophys 463: 149-167, 2007.

16. Holloway ZG, Velayos-Baeza A, Howell GJ, Levecque C, Ponnambalam S, Sztul E and Monaco AP: Trafficking of the Menkes copper transporter ATP7A is regulated by clathrin-, AP-2-, AP-1-, and Rab22-dependent steps. Mol Biol Cell 24: 1735-1748, S1-S8, 2013.

17. McKie AT and Barlow DJ: The SLC40 basolateral iron transporter family (IREG1/ferroportin/MTP1). Pflugers Arch 447: 801-806, 2004.

18. Miret S, Simpson RJ and McKie AT: Physiology and molecular biology of dietary iron absorption. Annu Rev Nutr 23: 283-301, 2003 .

19. Aguirre P, Mena N, Tapia V, Arredondo M and Núñez MT: Iron homeostasis in neuronal cells: A role for IREG1. BMC Neurosci 6: 3, 2005 .

20. Kelleher T, Ryan E, Barrett S, Sweeney M, Byrnes V, O'Keane C and Crowe J: Increased DMT1 but not IREG1 or HFE mRNA following iron depletion therapy in hereditary haemochromatosis. Gut 53: 1174-1179, 2004.

21. Yeh KY, Yeh M, Mims L and Glass J: Iron feeding induces ferroportin 1 and hephaestin migration and interaction in rat duodenal epithelium. Am J Physiol Gastrointest Liver Physiol 296: G55-G65, 2009.

22. Lee SM, Attieh ZK, Son HS, Chen H, Bacouri-Haidar M and Vulpe CD: Iron repletion relocalizes hephaestin to a proximal basolateral compartment in polarized MDCK and Caco2 cells. Biochem Biophys Res Commun 421: 449-455, 2012.

23. Fuqua BK, Lu Y, Darshan D, Frazer DM, Wilkins SJ, Wolkow N, Bell AG, Hsu J, Yu CC, Chen H, et al: The multicopper ferroxidase hephaestin enhances intestinal iron absorption in mice. PLoS One 9: e98792, 2014.

24. Gaggelli E, Kozlowski H, Valensin D and Valensin G: Copper homeostasis and neurodegenerative disorders (Alzheimer's, prion, and Parkinson's diseases and amyotrophic lateral sclerosis). Chem Rev 106: 1995-2044, 2006. 
25. Hubner CA and Jentsch TJ: Ion channel diseases. Hum Mol Genet 11: 2435-2445, 2002.

26. Hudson DM, Curtis SB, Smith VC, Griffiths TA, Wong AY Scudamore $\mathrm{CH}$, Buchan AM and MacGillivray RT: Human hephaestin expression is not limited to enterocytes of the gastrointestinal tract but is also found in the antrum, the enteric nervous system, and pancreatic \{beta\}-cells. Am J Physiol Gastrointest Liver Physiol 298: G425-G432, 2010.

27. Lee GS, Choi KC and Jeung EB: K+-dependent $\mathrm{Na}+/ \mathrm{Ca} 2+$ exchanger 3 is involved in renal active calcium transport and is differentially expressed in the mouse kidney. Am J Physiol Renal Physiol 297: F371-F379, 2009.

28. Nose Y, Wood LK, Kim BE, Prohaska JR, Fry RS, Spears JW and Thiele DJ: Ctrl is an apical copper transporter in mammalian intestinal epithelial cells in vivo that is controlled at the leve of protein stability. J Biol Chem 285: 32385-32392, 2010.

29. White C, Lee J, Kambe T, Fritsche K and Petris MJ: A role for the ATP7A copper-transporting ATPase in macrophage bactericidal activity. J Biol Chem 284: 33949-33956, 2009.

30. Kuo YM, Gybina AA, Pyatskowit JW, Gitschier J and Prohaska JR: Copper transport protein (Ctr1) levels in mice are tissue specific and dependent on copper status. J Nutr 136: 21-26, 2006.

31. Vennekens R, Owsianik G and Nilius B: Vanilloid transient receptor potential cation channels: An overview. Curr Pharm Des 14: 18-31, 2008

32. Wolff NA, Liu W, Fenton RA, Lee WK, Thévenod F and Smith CP: Ferroportin 1 is expressed basolaterally in rat kidney proximal tubule cells and iron excess increases its membrane trafficking. J Cell Mol Med 15: 209-219, 2011.

33. Tanner MR and Beeton C: Differences in ion channel phenotype and function between humans and animal models. Front Biosci (Landmark Ed) 23: 43-64, 2018.

34. Lin JH: Species similarities and differences in pharmacokinetics. Drug Metab Dispos 23: 1008-1021, 1995.

35. Chandler K: Canine epilepsy: What can we learn from human seizure disorders? Vet J 172: 207-217, 2006.

36. Livak KJ and Schmittgen TD: Analysis of relative gene expression data using real-time quantitative PCR and the 2(-Delta Delta C(T)) method. Methods 25: 402-408, 2001

37. Entin-Meer M, Levy R, Goryainov P, Landa N, Barshack I, Avivi C, Semo J and Keren G: The transient receptor potential vanilloid 2 cation channel is abundant in macrophages accumulating at the peri-infarct zone and may enhance their migration capacity towards injured cardiomyocytes following myocardial infarction. PLoS One 9: e105055, 2014.

38. Kiela PR and Ghishan FK: Physiology of intestinal absorption and secretion. Best Pract Res Clin Gastroenterol 30: 145-159, 2016.

39. Kulbacka J, Choromańska A, Rossowska J, Weżgowiec J, Saczko J and Rols MP: Cell membrane transport mechanisms: Ion channels and electrical properties of cell membranes. Adv Anat Embryol Cell Biol 227: 39-58, 2017.

40. Santoni G, Farfariello V, Liberati S, Morelli MB, Nabissi M, Santoni M and Amantini C: The role of transient receptor potential vanilloid type-2 ion channels in innate and adaptive immune responses. Front Immunol 4: 34, 2013.

41. Yang H, An BS, Choi KC and Jeung EB: Change of genes in calcium transport channels caused by hypoxic stress in the placenta, duodenum, and kidney of pregnant rats. Biol Reprod 88 $30,2013$.
42. Kruger MC and Wolber FM: Osteoporosis: Modern paradigms for last century's bones. Nutrients 8: pii: E376, 2016.

43. Entin-Meer M, Cohen L, Hertzberg-Bigelman E, Levy R, Ben-Shoshan J and Keren G: TRPV2 knockout mice demonstrate an improved cardiac performance following myocardial infarction due to attenuated activity of peri-infarct macrophages. PLoS One 12: e0177132, 2017

44. Hassan S, Eldeeb K, Millns PJ, Bennett AJ, Alexander SP and Kendall DA: Cannabidiol enhances microglial phagocytosis via transient receptor potential (TRP) channel activation. Br J Pharmacol 171: 2426-2439, 2014.

45. Link TM, Park U, Vonakis BM, Raben DM, Soloski MJ and Caterina MJ: TRPV2 has a pivotal role in macrophage particle binding and phagocytosis. Nat Immunol 11: 232-239, 2010.

46. Yamashiro K, Sasano T, Tojo K, Namekata I, Kurokawa J, Sawada N, Suganami T, Kamei Y, Tanaka H, Tajima N, et al: Role of transient receptor potential vanilloid 2 in LPS-induced cytokine production in macrophages. Biochem Biophys Res Commun 398: 284-289, 2010.

47. Holzer P: TRP channels in the digestive system. Curr Pharm Biotechnol 12: 24-34, 2011.

48. Sulk M, Seeliger S, Aubert J, Schwab VD, Cevikbas F, Rivier M, Nowak P, Voegel JJ, Buddenkotte J and Steinhoff M: Distribution and expression of non-neuronal transient receptor potential (TRPV) ion channels in rosacea. J Invest Dermatol 132: 1253-1262, 2012.

49. Ohrvik H and Thiele DJ: How copper traverses cellular membranes through the mammalian copper transporter 1, Ctr1. Ann N Y Acad Sci 1314: 32-41, 2014.

50. Collins JF, Hua P, Lu Y and Ranganathan PN: Alternative splicing of the Menkes copper Atpase (Atp7a) transcript in the rat intestinal epithelium. Am J Physiol Gastrointest Liver Physiol 297: G695-G707, 2009.

51. Linz R, Barnes NL, Zimnicka AM, Kaplan JH, Eipper B and Lutsenko S: Intracellular targeting of copper-transporting ATPase ATP7A in a normal and Atp7b-/-kidney. Am J Physiol Renal Physiol 294: F53-F61, 2008.

52. Vonk WI, de Bie P, Wichers CG, van den Berghe PV, van der Plaats R, Berger R, Wijmenga C, Klomp LW and van de Sluis B: The copper-transporting capacity of ATP7A mutants associated with Menkes disease is ameliorated by COMMD1 as a result of improved protein expression. Cell Mol Life Sci 69: 149-163, 2012.

53. Pinnix ZK, Miller LD, Wang W, D'Agostino R Jr, Kute T, Willingham MC, Hatcher H, Tesfay L, Sui G, Di X, et al: Ferroportin and iron regulation in breast cancer progression and prognosis. Sci Transl Med 2: 43ra56, 2010.

54. Kong WN, Chang YZ, Wang SM, Zhai XL, Shang JX, Li LX and Duan XL: Effect of erythropoietin on hepcidin, DMT1 with IRE, and hephaestin gene expression in duodenum of rats. J Gastroenterol 43: 136-143, 2008.

55. Petrak J and Vyoral D: Hephaestin-a ferroxidase of cellular iron export. Int J Biochem Cell Biol 37: 1173-1178, 2005.

56. Malik IA, Naz N, Sheikh N, Khan S, Moriconi F, Blaschke M and Ramadori G: Comparison of changes in gene expression of transferrin receptor-1 and other iron-regulatory proteins in rat liver and brain during acute-phase response. Cell Tissue Res 344: 299-312, 2011. 